\title{
Liebe Leserin, lieber Leser
}

Die gute Zusammenarbeit der sogenannten Partner im Gesundheitswesen wird viel beschworen, aber selten realisiert. In dieser Ausgabe der SÄZ können wir für einmal ein Gegenbeispiel präsentieren. Der «Kommentar zum Vorgehen bei Rückrufen der Hersteller im Falle potentieller Fehlfunktionen von kardialen Implantaten» ist beeindruckend breit abgestützt. Das von verschiedenen medizinischen Fachgesellschaften, swissmedic und Vertretern der Industrie sowie deren Dachorganisation FASMED verfasste Papier wird auch von santésuisse, dem Dachverband der Schweizerischen Patientenstellen, der Schweizerischen Gesellschaft für Kardiologie und der FMH mitgetragen. Hanspeter Kuhn, Leiter des Rechtsdienstes der FMH, würdigt die Gemeinschaftsleistung zudem im Editorial $(\rightarrow$ Seite 407 und $\rightarrow$ Seite 410 ).

Werner Bauer engagiert sich schon seit vielen Jahren für die Belange der Hausarztmedizin, unter anderem im Rahmen seiner standespolitischen Arbeit für die Schwei- zerische Gesellschaft für Innere Medizin SGIM und das Kollegium für Hausarztmedizin KHM. In beiden Organisationen spielte er als Präsident über Jahre eine prägende Rolle, in beiden Organisationen ist er weiterhin an vorderster Front präsent. Vor diesem Hintergrund ist gut nachvollziehbar, dass ihn die Veranstalter der Arbeitstagung Nationale Gesundheitspolitik vom November letzten Jahres einluden, den versammelten Experten seine Überlegungen zur Perspektive der Hausarztmedizin in der Schweiz zu präsentieren. Wir freuen uns darüber, Werner Bauers bemerkenswertes und unverändert aktuelles «Impulsreferat» nun auch in der SÄZ wiedergeben zu können ( $\rightarrow$ Seite 429 ).

Ein engagierter Mann ist auch der deutsche Kollege Wolfgang Starke: Er führt seit 2003 als Chefarzt das Amppipal Community Hospital im westlichen Nepal, das eine Region mit rund 200000 Einwohnern versorgt. Die Suche nach einem Nachfolger - in Nepal wird über 65jährigen keine
Arbeitserlaubnis mehr erteilt - gestaltet sich schwierig: Ein vor knapp zwei Jahren publizierter Aufruf im Deutschen Ärzteblatt blieb ohne Resonanz. Es wäre Starke zu wünschen, dass er in der Schweiz mehr Glück hat. Einen Einblick in seine ebenso vielseitige wie anspruchsvolle Arbeit gibt er $\mathrm{ab} \rightarrow$ Seite 435 .

Film, Film, Film: Kulturteil und Zu guter Letzt stehen für einmal ganz im Zeichen dieses Mediums. Erhard Taverna stellt eine ganze Reihe filmischer Auseinandersetzungen zum Stichwort "Störfall Jugend» sowie die feinfühlige Dokumentation des Sterbeprozesses eines HIV-infizierten Mannes vor. Bruno Kesseli geht anhand des Porträts eines französischen Allgemeinpraktikers der Frage nach, inwiefern ein solcher Film auch für Berufskolleginnen und -kollegen interessant sein kann $(\Leftrightarrow$ Seite 445,447 und $\Rightarrow$ 448).

Die Redaktion

\section{IMPRESSUM}

\section{Redaktion}

Dr. med. et lic. phil. Bruno Kesseli (Chefredaktor)

Daniel Lüthi, FMH

Dr. med. Erhard Taverna

Redaktion Ethik

Dr. theol. Christina Aus der Au Prof. Dr. med. Lazare Benaroyo Prof. Dr. med. Lazare Bena
Prof. Dr. phil., dipl. biol. Christoph Rehmann-Sutter

Redaktion Geschichte PD Dr. med. et lic. phil. Iris Ritzman Prof. Dr. med. Jean Jacques Dreifuss Dr. rer. soc. Eberhard Wolff

Managing Editor

Thomas Heuer, lic. phil

\section{Redaktionssekretariat}

Margrit Neff

Redaktionsadresse

EMH Schweizerischer Ärzteverlag AG Farnsburgerstrasse 8, 4132 Muttenz Tel. 06146785 55, Fax 0614678556 E-Mail: redaktion.saez@emh.ch Internet: www.saez.ch
Herausgeber

FMH

Verbindung der Schweizer Ärztinnen und Ärzte

Fédération des médecins suisses

Federazione dei medici svizzeri

Federazione dei medici svizs
Swiss

Swiss Medical Association

Elfenstrasse 18, 3000 Bern 15

E-Mail: info@fmh.ch

Internet: www.fmh.ch

Verlag

EMH Editores Medicorum Helveticorum

EMH Schweizerischer Ärzteverlag AG

EMH Editions médicales suisses SA

EMH Edizioni mediche svizzere SA

EMH Swiss Medical Publishers Ltd.

Postfach, 4010 Basel

Tel. 06146785 55, Fax 0614678556

E-Mail: verlag@emh.ch

E-Mail: verlag@emh.ch

Herstellung

Schwabe AG

Farnsburgerstrasse 8

Postfach 832, 4132 Muttenz

Tel. 06146785 85, Fax 0614678586

E-Mail: druckerei@schwabe.ch
Marketing EMH

EMH Schweizerischer Ärzteverlag AG Thomas Gierl M. A.

Leiter Marketing und Kommunikation Farnsburgerstrasse 8, 4132 Muttenz

Tel. 06146785 49, Fax 0614678556

E-Mail: tgierl@emh.ch

Inserate

EMH Schweizerischer Ärzteverlag AG

Ariane Furrer

Assistentin Inserateregi

Farnsburgerstrasse 8, 4132 Mutten

Tel. 0614678588

Fax 0614678556

E-Mail: afurrer@emh.ch

«Stellenmarkt»

EMH Schweizerischer Ärzteverlag AG

Farnsburgerstrasse 8, 4132 Muttenz

Tel. 06146785 55, Fax 0614678556

E-Mail: stellenmarkt@emh.ch

«Stellenvermittlung»

FMH Consulting Services

Stellenvermittlung

Postfach 246, 6208 Oberkirch

Tel. 04192500 77, Fax 0419210586

E-Mail: fmhstv@hin.ch

Internet: www.fmhjob.ch
Abonnemente

FMH-Mitglieder

FMH Verbindung der Schweizer

Ärztinnen und Ärzte

Elfenstrasse 18,3000 Bern 15

Tel. 03135911 11, Fax 0313591112

Andere Abonnemente

EMH Schweizerischer Ärzteverlag AG Abonnemente, Postfach, 4010 Basel Tel. 06146785 75, Fax 0614678576 E-Mail: abo@emh.ch

Jahresabonnement: CHF 320.-, zuzüglich Porto

(c) 2007 by EMH Schweizerischer Ärzteverlag AG, Basel. Alle Rechte vorbehalten. Nachdruck, elektronische Wiedergabe und Übersetzung, auch auszugsweise, nur mit schriftlicher Genehmigung des Verlages gestattet.

Erscheint jeden Mittwoch

ISSN 0036-7486

ISSN 1424-4004 (Elektronische Ausg.)
Delegierte der Fachgesellschaften Allergologie und Immunologie: Prof. Dr. A. Bircher

Allgemeinmedizin: Dr. B. Kissling Anästhesiologie und Reanimation Prof. P. Ravussin

Angiologie: Dr. B. Amman-Vesti Arbeitsmedizin: Dr. B. Merz Chirurgie: Prof. Dr. M. Decurtins Dermatologie und Venerologie: PD Dr. S. Lautenschlager Endokrinologie und Diabetologie: Prof. Dr. G.A. Spinas Prof. Dr. G.A. Spinas Geriatrie: Dr. M. Conzelmann Gynäkologie und Geburtshilfe:
Prof. Dr. Dr. h. c. mult. W. Holzgreve
Hämatologie: Dr. M. Zoppi Handchirurgie: PD Dr. L. Nagy Infektologie: Prof. Dr. W. Zimmerli Innere Medizin: Dr. W. Bauer Intensivmedizin: Dr. C. Jenni Kardiologie: Prof. Dr. B. Meier Kiefer- und Gesichtschirurgie: Dr. C. Schotland

Kinder- und Jugendpsychiatrie: Dr. R. Hotz Kinderchirurgie: Dr. M. Bittel

Medizinische Genetik: Prof. Dr. P. Miny

Neonatologie: Prof. Dr. H.-U. Bucher

Nephrologie: Prof. Dr. J.-P. Guignard

Neurochirurgie: Prof. Dr. H. Landolt

Neurochirurgie: Prof. Dr. H. Lando

Neuropädiatrie: Prof. Dr. J. Lütschg

Neuropadiatrie: Prof. Dr. J. Lütschg
Neuroradiologie: Prof. Dr. W. Wichmann
Nuklearmedizin: Prof. Dr. J. Mülle Onkologie: PD Dr. B. Pestalozzi Ophthalmologie: Dr. A. Franceschetti ORL, Hals- und Gesichtschirurgie: Prof. Dr. J.-P. Guyot

Orthopädie: Dr. T. Böni

Pädiatrie: Dr. R. Tabin

Pathologie: Prof. Dr. G. Cathomas

Pharmakologie und Toxikologie:

Dr. T. Buclin

Pharmazeutische Medizin: Dr. P. Kleist

Physikalische Medizin und Rehabilitation:

Dr. M. Weber

Dr. M. Weber
Plast.-Rekonstrukt. u. Ästhetische Chirurgie:

Prof. Dr. G. Pierer

Pneumologie: Prof. Dr. E. Russi
Prävention und Gesundheitswesen: Dr. C. Junker

Psychiatrie und Psychotherapie: Dr. G. Ebner

Radiologie: Prof. Dr. B. Marincek Radioonkologie: Prof. Dr. R. H. Greiner Rechtsmedizin: Prof. T. Krompeche Rheumatologie: Prof. Dr. M. Seitz Thorax-, Herz- und Gefässchirurgie: Prof. Dr. T. Carrel

Tropen- und Reisemedizin: PD Dr. C. Hatz Urologie: PD Dr. T. Zellweger 